C. A. Johnson

Nagoya Math. J.

Vol. 106 (1987), 91-100

\title{
DISTRIBUTIVE AND RELATED IDEALS IN GENERIC EXTENSIONS
}

\author{
C. A. JOHNSON
}

\section{§ 0. Introduction}

Let $\kappa$ be a regular uncountable cardinal and $I$ a $\kappa$-complete ideal on $\kappa$. In [11] Kanai proved that the $\mu$-distributivity of the quotient algebra $P(\kappa) / I$ is preserved under $\kappa$-c.c. $\mu$-closed forcing. In this paper we extend Kanai's result and also prove similar preservation results for other naturally occurring forms of distributivity. We also consider the preservation of two game theoretic properties of $I$ and in particular, using a game theoretic equivalent of precipitousness we give a new proof of Kakuda's theorem ([10]) that the precipitousness of $I$ is preserved under $\kappa$-c.c. forcing.

Our set theoretical notation and terminology is reasonably standard. Throughout $\kappa$ will denote a regular uncountable cardinal, $I$ a proper nonprincipal $\kappa$-complete ideal on $\kappa$ (in the sense of $[2$, p. 7]), and $P$ a forcing notion. We will only be interested in the case when $\|_{\bar{P}}$ " $\kappa$ is regular and $I$ generates an ideal on $\kappa$ ", and hence throughout we assume that $\|_{\bar{P}}$ " $\forall x$ (if $x \subseteq V$ and $|x|<\kappa$ then there exists a $y \in V$ such that $|y|^{V}<\kappa$ and $x \subseteq y) "$. $J$ will denote the $P$ name for the ideal on $\kappa$ generated by $I$.

Definition. $P$ is $(\mu, \eta, \lambda)$-distributive iff whenever $p \in P$ and $\left\langle W_{\alpha} \mid \alpha<\mu\right\rangle$ is a sequence of maximal antichains below $p$ in $P$, each of cardinality $\leqslant \lambda$, there is a $q \in P$ such that $q \leqslant p$ and for each $\alpha<\mu, \mid\left\{r \in W_{\alpha} \mid r\right.$ is compatible with $q\} \mid \leqslant \eta . \quad P$ is said to be $(\mu, \eta, \infty)$-distributive if it is $(\mu, \eta, \lambda)$ distributive for each $\lambda$. We omit the $\eta$ in the case $\eta=1$.

$I$ is said to be $(\mu, \eta, \lambda)$-distributive iff the Boolean algebra $P(\kappa) / I$ is $(\mu, \eta, \lambda)$-distributive.

\section{$\S 1$. $(\mu, \kappa, \infty)$ and $(\mu, \infty)$-distributivity}

In this section we make use of generic ultrafilters to prove some preservation results for $(\mu, \kappa, \infty)$ and $(\mu, \infty)$-distributivity.

Received October 16, 1985. 
Recall that $R(I)$ is the forcing notion whose conditions are sets $A \in I^{+}$ ( $=P(\kappa)-I$ ) with $A \leqslant A^{\prime}$ iff $A \subseteq A^{\prime}$. If $D$ is $R(I)$ - $V$-generic (where $V$ is our ground model), then $D$ is an ultrafilter on $P(\kappa) \cap V$ extending $I^{*}$, the filter dual to $I$, and within $V(D)$ we may form the ultrapower $V^{x} / D$ (see [6]). I is said to be precipitous iff $\|_{\overline{R(I)}}$ " $V / \dot{D}$ is well founded", and in this case we identify $V^{x} / D$ with its transitive collapse.

$(\mu, \kappa, \infty)$ and $(\mu, \infty)$-distributivity are related to $R(I)$ by the following

Theorem 1.1 (a) ([8]). For $\mu \leqslant \kappa, I$ is $(\mu, \kappa, \infty)$-distributive iff $\|_{R(I)}$ " $\left(V^{\kappa} / \dot{D}\right)^{\mu} \subseteq V^{\kappa} / \dot{D} "$ "

(b) I is $(\mu, \infty)$-distributive iff $\|_{\overline{R(I)}}$ " $V^{\mu} \subseteq V$ ".

Corollary 1.2. If $I$ is $(\omega, \kappa, \infty)$-distributive then $I$ is precipitous.

Let $j$ denote the natural embedding $j: V \rightarrow V^{k} / D$, then Baumgartner and Taylor [1] defined $P$ to be $I$-regular iff $\|_{\overline{R(I)}} " \forall p \in P \quad \exists q \in j(P)(q \leqslant j(p)$ and $q \|_{j(P)} j^{-1}(\dot{H})$ is $P$-V-generic)", where $\dot{H}$ denotes the canonical name for the $j(P)-V(D)$-generic set. They also proved the following.

LemMa 1.3 ([1, p. 603]). If $P$ has the $\kappa$-chain condition then $\left\|_{\overline{R(\bar{I})}}{ }^{e}\right\|_{\bar{\jmath} \overline{(P)}}$ $j^{-1}(\dot{H})$ is $P$-V-generic".

TheOREm $1.4\left([1 \text {, Theorem 5.2] })_{H}\right.$. Suppose $I$ is precipitous and $P$ is $I$ regular. If $D$ is $R(I)$-V-generic, $H$ is $j(P)-V(D)$-generic and $G=j^{-1}(H)$ is $P$ - $V$-generic, then in $V(D)(H), j: V \rightarrow V^{k} / D$ may be extended to an elementary embedding $\bar{j}: V(G) \rightarrow V^{\kappa} / D(H)$ such that $U=\{X \in P(\kappa) \cap V(G) \mid[\mathrm{id}] \in \bar{j}(X)\}$ is $R\left(J_{G}\right)-V(G)$-generic (where [id] denotes the equivalence class of the identity function in $\left.V^{x} / D\right)$.

$\bar{j}$ is given by $\bar{j}\left(\dot{x}_{G}\right)=(j(\dot{x}))_{H}$. Moreover it is easy to check that $V(G)^{x} / U$ $\cong V^{x} / D(H)$ and $V(D)(H)=V(G)(U)$.

Now for our preservation results.

Theorem 1.5. Suppose $\omega \leqslant \mu \leqslant \kappa, P$ has the $\kappa$-chain condition and $I$ is $(\mu, \kappa, \infty)$-distributive. If $\|_{\overline{R(I)}}$ " $j(P)$ is $(\mu, \mu, \infty)$-distributive", then $\|_{\bar{P}}$ " $J$ is $(\mu, \kappa, \infty)$-distributive".

Proof. Suppose $p \in P$ and $\dot{X}$ is $P$ name in $V$ such that $p \|_{P}{ }^{"}$ $\dot{X} \in J^{+}$ and $\dot{X} \|_{\overline{R(J)}}\left(V(\dot{G})^{x} / \dot{U}\right)^{\mu} \not Z V(\dot{G})^{x} / \dot{U}$ ". Let $E=\left\{\alpha<\kappa \mid \exists q \in P\left(q \leqslant p\right.\right.$ and $q \|_{\bar{P}}$ " $\alpha \in \dot{X} ")\}$, then $(E \in V), p \|_{P} " \dot{X} \subseteq E "$, hence $E \in I^{+}$and we may find a sequence $\left\langle q^{\alpha} \mid \alpha \in E\right\rangle \in V$ such that for each $\alpha \in E, q^{\alpha} \leqslant p$ and $q^{\alpha} \|_{\bar{P}}$ "e $\alpha \in \dot{X}$ ".

Let $D$ be $R(I)$ - $V$-generic with $E \in D$ and $H$ be $j(P)-V(D)$-generic with 
$\left[\left\langle q^{\alpha} \mid \alpha \in E\right\rangle\right] \in H$. By Lemma 1.3, $G=j^{-1}(H)$ is $P$ - $V$-generic and, since $V^{\alpha}|D|=$ " $\left[\left\langle q^{\alpha} \mid \alpha \in E\right\rangle\right] \|_{j_{j(P)}}[\mathrm{id}] \in j(\dot{X})$ ", if $U$ is the $R\left(J_{G}\right)-V(G)$-generic ultrafilter given in Theorem 1.4 then $\dot{X}_{G} \in U$. Also, since $j(p) \geqslant\left[\left\langle q^{\alpha} \mid \alpha \in E\right\rangle\right], p \in G$, hence by our hypothesis $\left(V(G)^{x} / U\right)^{\mu} \not \subset V(G)^{k} / U$ (and so $\left(V^{k} / D(H)\right)^{\mu} \not \subset V^{x} / D(H)$ ).

Therefore in $V(D)$ now we may find an $r \in H$ and a $j(P)$ name $\dot{f}$ such that $r \|_{\overline{j(P)}} " \dot{f}: \mu \rightarrow V^{\kappa} / D(\dot{H})$ and $\dot{f} \notin V^{\kappa} / D(\dot{H}) "$. Clearly we may assume that $\dot{f}$ is of the form $\left.\dot{f}=\left\langle\left\langle\alpha, x_{\alpha}^{\delta}\right\rangle, s_{\alpha}^{\delta}\right\rangle \mid \alpha<\mu, \delta<\delta_{\alpha}\right\}$ where for each $\alpha<\mu,\left\{s_{\alpha}^{\delta} \mid \delta<\delta_{\alpha}\right\}$ is a maximal antichain below $r$ in $j(P)$ and for each $\delta<\delta_{\alpha}, x_{\alpha}^{\delta}$ is a $j(P)$ name in $V^{*} / D$ (and $\left\langle\alpha, x_{\alpha}^{\delta}\right\rangle$ is the $j(P)$ name such that $\left.\|_{j(P)}\left\langle\alpha, x_{\alpha}^{\delta}\right\rangle_{\dot{H}}=\left\langle\alpha,\left(x_{\alpha}^{\delta}\right)_{\dot{H}}\right\rangle\right)$. Since $j(P)$ is $(\mu, \mu, \infty)$-distributive there is a $t \in j(P)$ such that $t \leqslant r$ and for each $\alpha<\mu, B_{\alpha}=\left\{\delta<\delta_{\alpha} \mid t\right.$ is compatible with $\left.s_{\alpha}^{\delta}\right\}$ has cardinality $\leqslant \mu$. Hence if we let $\dot{g}=\left\{\left\langle\left\langle\alpha, x_{\alpha}^{\delta}\right\rangle, s_{\alpha}^{\delta}\right\rangle \mid \alpha<\mu, \delta \in B_{\alpha}\right\}$, then by Theorem 1.1, $\dot{g} \in V^{\star} / D$ and clearly $t \|_{\frac{\partial(P)}{(P)}} " \dot{f}=\dot{g} \in V^{*} / D(\dot{H}) "$, contradicting $t \leqslant r$.

Theorem 1.5 may also be proved using the (well known) fact that $I$ is $(\mu, \kappa, \infty)$-distributive iff for each ordinal $\lambda$, $\|_{\overline{R(L)}}$ "for each $f$, if $f: \mu \rightarrow \lambda$ then there is a function $g: \mu \rightarrow P(\lambda)$ such that $g \in V$ and for each $\alpha<\mu$, $|g(\alpha)|^{v} \leqslant \kappa$ and $f(\alpha) \in g(\alpha) "$.

Under certain circumstances the converse of Theorem 1.5 also holds.

Theorem 1.6. Suppose $\kappa=\mu^{+}, P$ has the $\kappa$-chain condition and $I$ is $(\mu, \kappa, \infty)$-distributive. If $\|_{\bar{P}}$ "J is $(\mu, \kappa, \infty)$-distributive", then $\|_{\bar{R}(\bar{T})}$ " $j(P)$ is $(\mu, \mu, \infty)$-distributive".

Proof. Let $D$ be $R(I)-V$-generic. By Lemma 1.3 and Theorem 1.4, if $H$ is $j(P)$ - $V(D)$-generic then $G=j^{-1}(H)$ is $\rho$ - $V$-generic, $V(D)(H)=V(G)(U)$ and $V^{*} / D(H) \cong V(G)^{*} / U$ for some $R\left(J_{G}\right)-V(G)$-generic ultrafilter $U$, and since $J_{G}$ is $(\mu, \kappa, \infty)$-distributive, $\left(V^{*} / D(H)\right)^{\mu} \subseteq V^{*} / D(H)$.

In $V(D)$ now, suppose $r \in j(P)$ and $\left\langle W_{\alpha} \mid \alpha<\mu\right\rangle$ is a sequence of maximal antichains below $r$ in $j(P)$. Let $h: \bigcup\left\{W_{\alpha} \mid \alpha<\mu\right\} \rightarrow$ Ord be injective and $\dot{f}$ be the $j(P)$ name given by $\dot{f}=\left\{\langle\langle\alpha, h(q)\rangle, q\rangle \mid \alpha<\mu, q \in W_{\alpha}\right\}$. Then $r \|_{j(\bar{P})}$ " $\dot{f}: \mu \rightarrow$ Ord", and hence by our argument above there is a $t \in j(P)$ and a $j(P)$ name $\dot{g} \in V^{\kappa} / D$ such that $t \leqslant r$ and $t \|_{j(P)}$ " $\dot{f}=\dot{g} "$. As in Theorem 1.5 we may assume $\dot{g}$ to be of the form $\dot{g}=\left\{\left\langle\left\langle\alpha, \psi_{\alpha}^{o}\right\rangle, s_{\alpha}^{o}\right\rangle \mid \alpha<\mu, \delta<\delta_{\alpha}\right\}$ where for each $\alpha<\mu,\left\{s_{\alpha}^{\delta} \mid \delta<\delta_{\alpha}\right\}$ is a maximal antichain below $t$ in $j(P)$ and for each $\delta<\delta_{\alpha}, \psi_{\alpha}^{\delta}$ is an ordinal.

Suppose $\alpha<\mu$, then $\left\{s_{\alpha}^{\delta} \mid \delta<\delta_{\alpha}\right\} \in V^{*} / D$ and so, since $P$ has the $\kappa$-chain condition and $j$ is elementary, $\left|\delta_{\alpha}\right| \leqslant j(\mu)=\mu$. Also if $q \in W_{\alpha}$ is compatible with $t$, there exists a $\delta<\delta_{\alpha}$ such that $q$ is compatible with $s_{\alpha}^{\delta}$, and hence 
since $q \|_{\overline{j(P)}} " \dot{f}(\alpha)=h(q) "$ and $s_{\alpha}^{\hat{o}} \|_{\overline{j(\bar{P})}} " \dot{f}(\alpha)=\dot{g}(\alpha)=\psi_{\alpha}^{\hat{o}} "$, we must have $h(q)=\psi_{\alpha}^{\delta}$. Thus $\mid\left\{q \in W_{\alpha} \mid q\right.$ is compatible with $\left.t\right\} \mid \leqslant \mu$.

In [1] Baumgartner and Taylor defined an ideal $I$ on $\omega_{1}$ to be presaturated iff $I$ is $\omega_{2}$-preserving (i.e. $\|_{\overline{R(I)}}$ " $\omega_{2}^{V}$ is a cardinal") and precipitous. It is clear that if $I$ is an $\left(\omega, \omega_{1}, \infty\right)$-distributive ideal on $\omega_{1}$, then $I$ is presaturated (since $\|_{\bar{R}(\bar{I})} "\left|\omega_{1}^{V}\right|=\omega "$, see [2, p. 52]) and that the converse holds for $\omega_{3}$-saturated ideals (see [1, Theorem 4.2]).

Baumgartner and Taylor also proved ([1, Theorem 5.10]) that presaturation is a strictly weaker notion than $\omega_{2}$-saturation in that if $P$ is the forcing notion for adding a closed unbounded subset of $\omega_{1}$ with finite conditions (i.e. $p \in P$ iff $p$ is a finite function mapping $\omega_{1}$ into $\omega_{1}$ for which there is a countable closed set $C \subseteq \omega_{1}$ so that if $h$ enumerates $C$ in increasing order, then $p \subseteq h ; p \leqslant p^{\prime}$ iff $p \supseteq p^{\prime}$ ) then $\|_{\bar{P}}{ }^{\text {" }} \omega_{1}^{V}=\omega_{1}$ and $\omega_{1}$ carries no $\omega_{2}$-saturated ideals", and if $I$ is a presaturated ideal on $\omega_{1}$ then $\|_{\bar{P}}$ " $\exists X \in J^{+}$, $J \mid X$ is presaturated". Using Theorem 1.5, a similar argument yields an analogous result for $\left(\omega, \omega_{1}, \infty\right)$-distributivity.

Theorem 1.7. If $I$ is an $\left(\omega, \omega_{1}, \infty\right)$-distributive ideal on $\omega_{1}$ and $P$ is the forcing notion for adding a closed unbounded subset of $\omega_{1}$ with finite conditions, then $\|_{P^{-}}{ }^{\prime} \exists X \in J^{+}, J \mid X$ is $\left(\omega, \omega_{1}, \infty\right)$-distributive".

Proof. Firstly note that since $|P|=\omega_{1}$ and forcing with $P$ does not collapse $\omega_{1}, P$ is $(\omega, \omega, \infty)$-distributive. Suppose $p \in P$ is such that $p \|_{\bar{P}}$ " $\forall X \in J^{+}, J \mid X$ is not $\left(\omega, \omega_{1}, \infty\right)$-distributive". In [1, Theorem 5.8] it is shown that $P$ is $I$-regular, hence if $D$ is $R(I)$ - $V$-generic we may find a $j(P)-V(D)$ generic set $H$ such that $G=j^{-1}(H)$ is $P$-V-generic and $p \in G$. It is easy to see that $j(P)$ is $P$ as defined in $V(D)$, hence $j(P)$ is $(\omega, \omega, \infty)$-distributive and as in Theorem 1.5 there is in $V(D)(H)$ an $R\left(J_{G}\right)-V(G)$-generic ultrafilter $U$ such that $\left(V(G)^{\omega_{1}} / U\right)^{\omega} \subseteq V(G)^{\omega_{1}} / U$. Therefore we may find an $X \in J_{G}^{+}$forcing this statement, and hence $J_{G} \mid X$ is $\left(\omega, \omega_{1}, \infty\right)$-distributive, contradicting $p \in G$.

CoRollary 1.8. If $Z F C+"{ }^{~} \omega_{1}$ carries an $\left(\omega, \omega_{1}, \infty\right)$-distributive ideal" is consistent, then so is $Z F C+{ }^{" ~} \omega_{1}$ carries an $\left(\omega, \omega_{1}, \infty\right)$-distributive ideal but no $\omega_{2}$-saturated ideals".

It is clear that our arguments in Theorem 1.7 also yield the following.

TheOREM 1.9. Suppose $\mu \leqslant \kappa, P$ is I-regular and $I$ is $(\mu, \kappa, \infty)$-distributive. If $\|_{\overline{R(I)}} " j(P)$ is $(\mu, \mu, \infty)$-distributive", then $\|_{\bar{P}}{ }^{"} \exists X \in J^{+}, J \mid X$ is 
$(\mu, \kappa, \infty)$-distributive".

In [11] Kanai proved that if $\mu<\kappa, I$ is $(\mu, \infty)$-distributive and $P$ has the $\kappa$-chain condition and is $\mu$-closed then $\|_{\bar{P}}$ " $J$ is $(\mu, \infty)$-distributive". Clearly under these conditions $\|_{\overline{R(I)}}$ " $j(P)$ is $\mu$-closed", and hence arguments similar to those of Theorems 1.5 and 1.7 easily yield the following strengthening of Kanai's result whose proof we leave to the reader.

Theorem 1.10. Suppose $\mu \leqslant \kappa, P$ is I-regular and $I$ is $(\mu, \infty)$-distributive. If $\|_{\overline{R(I)}} " J(P)$ is $(\mu, \infty)$-distributive", then $\|_{\bar{P}} " \exists X \in J^{+}, J \mid X$ is $(\mu, \infty)$-distributive". Moreover if $P$ has the $\kappa$-chain condition then we may take $X=\kappa$.

In the case when $|P|<\kappa$ we can do slightly better.

Theorem 1.11. Suppose, $\omega \leqslant \mu \leqslant \kappa,|P|<\kappa$ and I is $(\mu, \infty)$-distributive. If $\|_{\overline{R(I)}}$ "J $J(P)$ is $(\mu, \mu, \infty)$-distributive", then $\|_{\bar{P}}$ "J is $(\mu, \infty)$-distributive".

Proof. Suppose $p \in P$ and $\dot{X}$ is a $P$ name such that $p \|_{-}$" $\dot{X} \in J^{+}$and $\dot{X} \|_{\mid \overline{R(J)}} \exists f: \mu \rightarrow$ Ord, $f \notin V(\dot{G})$ ". As in Theorem 1.5 we may find an $R(I)-V$ generic set $D$ and a $j(P)-V(D)$-generic set $H$ such that $G=j^{-1}(H)$ is $P-V$ generic and $V(D)(H)$ contains a function $f: \mu \rightarrow$ Ord with $f \notin V(G)$.

Hence in $V(D)$ now we may and an $r \in H$ and a $j(P)$ name $\dot{f}$ such that $r \|_{\overline{j(P)}}$ " $\dot{f}: \mu \rightarrow$ Ord and $\dot{f} \notin V\left(j^{-1}(\dot{H})\right) "$. As in Theorem 1.5 again we may find a $t \leqslant r$ and a $j(P)$ name $\dot{g}=\left\{\left\langle\left\langle\alpha, \psi_{\alpha}^{o}\right\rangle, s_{\alpha}^{o}\right\rangle \mid \alpha<\mu, \delta<\beta_{\alpha}\right\}$ such that for each $\alpha<\mu, \beta_{\alpha} \leqslant \mu$ and $t \|_{\overline{j(P)}}$ " $\dot{f}=\dot{g} "$. Since $|P|<\kappa, j(P)=\{j(p) \mid p \in P\}$, hence let $\dot{h}=\left\{\left\langle\left\langle\alpha, \psi_{\alpha}^{\delta}\right\rangle, q_{\alpha}^{\delta}\right\rangle \mid \alpha<\mu, \delta<\beta_{\alpha}\right\}$ where $j\left(q_{\alpha}^{\delta}\right)=s_{\alpha}^{\delta}$ for each $\alpha<\mu$, $\delta<\beta_{\alpha}$. By $(\mu, \infty)$-distributivity of $I, \dot{h}$ is a $P$ name in $V$ and hence $t \|_{\overline{j(P)}} " \dot{f}_{\dot{H}}=\dot{g}_{\dot{H}}=\dot{h}_{j^{-1}(\dot{H})} \in V\left(j^{-1}(\dot{H})\right) "$, contradicting $t \leqslant r$.

\section{§ 2. $(\mu, \kappa)$-distributivity}

Our interest in $(\mu, \kappa)$-distributivity stems from its relationship with ideal theoretic partition relations (see [7]) and the following.

THEOREm 2.1 ([7]). For each cardinal $\mu<\kappa$ the following are equivalent:

(a) I is $(\mu, k)$-distributive

(b) $\|_{\overline{R(I)}}$ "for each $f \in V^{\star} / D$, if $f: \mu \rightarrow \kappa$ then $f \in V^{\prime}$

(c) $\forall \lambda<\kappa\left(\lambda^{\mu}<\kappa\right)$ and for every minimal unbounded I-function $h$, $\{\alpha \in \operatorname{dom}(h) \mid \operatorname{cf}(h(\alpha)) \leqslant \mu\} \in I$,

where $h: A \rightarrow \kappa$ is said to be a minimal unbounded I-function if $A \in I^{+}$, $h^{-1}(\{\rho\}) \in I$ for each $\rho<\kappa$, and whenever $f: B \rightarrow \kappa$ with $B \in P(A) \cap I^{+}$, either 
there exists a $\sigma<\kappa$ such that $f^{-1}(\{\sigma\}) \in I^{+}$or an $\alpha \in B$ such that $f(\alpha) \geqslant h(\alpha)$.

Theorem 2.2. Suppose $\mu$ is a cardinal, $\mu<\kappa$ and $I$ is $(\mu, \kappa)$-distributive. If $P$ has the $\kappa$-chain condition and is $(\mu, \alpha)$-distributive for each $\alpha<\kappa$, then $\|_{\bar{P}}$ "J is $(\mu, \kappa)$-distributive".

We will need the following

Lemma 2.3. Suppose $P$ has the $\kappa$-chain condition, $p \in P, E \in I^{+}$and $\left\{q^{\alpha} \mid \alpha \in E\right\} \subseteq P$ is such that for each $\alpha \in E, q^{\alpha} \leqslant p$. Then there is an $r \in P$ such that $r \leqslant p$ and $r \| \bar{P}$ " $\left\{\alpha \in E \mid q^{\alpha} \in \dot{G}\right\} \in J^{+} "$.

Proof. Suppose not, then $p \|_{\bar{P}}$ " $\{\alpha \in E \mid q \in \dot{G}\} \in J "$, and so we may find $\left\{r_{\delta} \mid \delta<\beta<\kappa\right\} \subseteq P$, a maximal antichain below $p$ in $P$ and $\left\{A_{\dot{\delta}} \mid \delta<\beta<\kappa\right\}$ $\subseteq I$ such that for each $\delta<\beta, r_{\delta} \|_{\bar{P}}{ }^{"}\left\{\alpha \in E \mid q^{\alpha} \in \dot{G}\right\} \subseteq A_{\delta}$ ". But then $A=$ $\bigcup\left\{A_{\delta} \mid \delta<\beta\right\} \in I, p \|_{T_{P}}{ }^{\prime \prime}\left\{\alpha \in E \mid q^{\alpha} \in \dot{G}\right\} \subseteq A "$, and hence if $\alpha \in E-A, p \|_{\bar{P}}$ " $q^{\alpha} \notin \dot{G} "$, contradicting the fact that $q^{\alpha} \leqslant p$.

Proof of Theorem 2.2. Using Theorem 2.1 (c), suppose $p \in P$ and $\dot{h}$ is a $P$ name such that $p \|_{\bar{P}}$ " $\dot{h}$ is a minimal unbounded $J$-function such that for each $\alpha \in \operatorname{dom}(\dot{h}), \operatorname{cf}(\dot{h}(\alpha)) \leqslant \mu$ ". As in Theorem 1.5 we may find an $E \in I^{+}$, a function $g: E \rightarrow \kappa$ and a sequence $\left\langle q^{\alpha} \mid \alpha \in E\right\rangle$ such that for each $\alpha \in E, q^{\alpha} \leqslant p$ and $q^{\alpha} \|_{T} " \alpha \in \operatorname{dom}(\dot{h})$ and $\dot{h}(\alpha)=g(\alpha) " . \quad P$ is clearly $(\mu, \infty)$ distributive hence, since $q^{\alpha} \|_{P}{ }^{\prime} \operatorname{cf}(g(\alpha)) \leqslant \mu$ ", we must have $\operatorname{cf}(g(\alpha)) \leqslant \mu$. We show that $g$ is a minimal unbounded $I$-function, contradicting the $(\mu, \kappa)$-distributivity of $I$.

Suppose $\rho<\kappa$ and $g^{-1}(\{\rho\}) \in I^{+}$, then by Lemma 2.3 we may find an $r \leqslant p$ such that $r \|_{\bar{P}}{ }^{" e}\left\{\alpha \in g^{-1}(\{\rho\}) \mid q^{\alpha} \in \dot{G}\right\} \in J^{+"}$ and hence $r \|_{\bar{P}}{ }^{~ "} \dot{h}^{-1}(\{\rho\}) \in$ $J^{+}$, a contradiction. Suppose $B \in P(E) \cap I^{+}$and $f: B \rightarrow \kappa$ is such that $f(\alpha)<g(\alpha)$ for each $\alpha \in B$. Again we may find an $s \leqslant p$ such that $s \|_{\bar{P}}$ " $\left\{\alpha \in B \mid q^{\alpha} \in \dot{G}\right\} \in J^{+} "$ and so $s \|_{\bar{P}}$ " $\{\alpha \in B \mid f(\alpha)<\dot{h}(\alpha)\} \in J^{+}$". Hence there is a $t \leqslant s$ and a $\sigma<\kappa$ such that $t \|_{\bar{P}^{-}} " f^{-1}(\{\sigma\}) \in J^{+} "$, thus $f^{-1}(\{\sigma\}) \in I^{+}$.

Finally by $(\mu, \infty)$-distributivity of $P$ and $(\mu, \kappa)$-distributivity of $I$, for each $\lambda<\kappa, \| \bar{P}{ }^{"} \lambda^{\mu}=\left(\lambda^{\mu}\right)^{V}<\kappa "$.

\section{§3. Precipitousness and games}

$G(I)$ is the following infinite game between two players, One and Two: Player One moves first and chooses a set $A_{0} \in I^{+}$. Then Two chooses a set $B_{0} \in P\left(A_{0}\right) \cap I^{+}$. One then chooses an $A_{1} \in P\left(B_{0}\right) \cap I^{+}$, and so on. Thus they produce a sequence 


$$
A_{0} \supseteq B_{0} \supseteq A_{1} \supseteq B_{1} \supseteq A_{2} \supseteq \cdots
$$

of sets in $I^{+}$, and Player One wins iff $\cap\left\{A_{n} \mid n<\omega\right\}=\emptyset$.

$G_{1}(I)$ is the game played as in $G(I)$ except that, in addition, for his $n^{\text {th }}$ move Player One also chooses a function $f_{n}: A_{n} \rightarrow$ Ord such that for each $n>0$ and $\alpha \in A_{n}, f_{n}(\alpha)<f_{n-1}(\alpha)$. If Player One can continue the play for $\omega$ moves, he wins; otherwise Two wins.

Theorem 3.1 ([4] and [5]). The following are equivalent:

(a) I is precipitous

(b) Player One does not have a winning strategy in $G(I)$

(c) Player One does not have a winning strategy in $G_{1}(I)$

(d) Player Two has a winning strategy in $G_{1}(I)$.

Using the game $G_{1}(I)$ we first give a new proof of the following theorem which is originally due to Kakuda ([10]) and (independently) Magidor ([12]).

THEOREM 3.2. If I is precipitous and $P$ has the $\kappa$-chain condition then $\|_{\bar{P}}$ "J $J$ is precipitous".

Proof. Firstly suppose $p \in P$ and $\dot{X}$ and $\dot{f}$ are $P$ names in $V$ such that $p \|_{P}$ " $\dot{X} \in J^{+}$and $\dot{f}: \dot{X} \rightarrow$ Ord". As in Theorem 1.5, given any $s \leqslant p$ we may find an $E \in I^{+}$, a function $g: E \rightarrow$ Ord $(g \in V)$ and a sequence $\left\langle q^{\alpha} \mid \alpha \in E\right\rangle \in V$ such that for each $\alpha \in E, q^{\alpha} \leqslant s$ and $q^{\alpha} \|_{P} " \alpha \in \dot{X}$ and $\dot{f}(\alpha)$ $=g(\alpha)$ ". Since $I$ is precipitous there is a $B \in P(E) \cap I^{+}$and an ordinal $\delta$ such that $B \|_{\overline{R(I)}} "[g]=\delta "$, and by Lemma 2.3 we may find an $r \leqslant s$ such that $r \|_{P} "\left\{\alpha \in B \mid q^{\alpha} \in \dot{G}\right\} \in J^{+} "$.

Suppose now that $G$ is $P$ - $V$-generic and in $V(G), S$ is a winning strategy for Player One in $G_{1}\left(J_{G}\right)$. By our argument above it is clear that we may construct a play of $G_{1}\left(J_{G}\right)$

$$
\left(X_{0}, f_{0}\right) \quad Y_{0}\left(X_{1}, f_{1}\right) \quad \ldots \quad\left(X_{n}, f_{n}\right) \quad Y_{n}\left(X_{n+1}, f_{n+1}\right) \quad \ldots
$$

in which Player One plays according to $S$ and such that for each $n<\omega$ there exist names $\dot{X}_{n}$ and $\dot{f}_{n}$ for $X_{n}$ and $f_{n}$, conditions $p_{n} \geqslant s_{n} \geqslant r_{n} \geqslant p_{n+1}$ $\in G, B_{n}, E_{n} \in I^{+}$(with $B_{n} \subseteq E_{n}$ ), $g_{n}: E_{n} \rightarrow$ Ord $\left(g_{n} \in V\right)$ and $\left\langle q_{n}^{\alpha} \mid \alpha \in E_{n}\right\rangle \in V$ satisfying:

(1) $p_{n} \|{ }_{\bar{P}}$ " $\dot{X}_{n} \in \mathcal{J}^{+}$and $\dot{f}_{n}: \dot{X}_{n} \rightarrow$ Ord"

(2) for each $\alpha \in E_{n}, q_{n}^{\alpha} \leqslant s_{n}$ and $q_{n}^{\alpha} \|_{\bar{P}}$ " $\alpha \in \dot{X}_{n}$ and $\dot{f}_{n}(\alpha)=g_{n}(\alpha)$ ",

(3) $B_{n} \|_{\bar{R}(I)}$ " $\left[g_{n}\right]=\delta_{n}$ " for some ordinal $\delta_{n}$ 
(4) $r_{n} \| \bar{P} "\left\{\alpha \in B_{n} \mid q_{n}^{\alpha} \in \dot{G}\right\} \in J^{+} "$

(5) $Y_{n}=\left\{\alpha \in B_{n} \mid q_{n}^{\alpha} \in G\right\}$ (and hence since $r_{n} \in G, Y_{n} \in P\left(X_{n}\right) \cap J^{+}$)

(6) if $n>0, p_{n} \|_{{ }_{P}}{ }^{~} \dot{X}_{n} \subseteq\left\{\alpha \in B_{n-1} \mid q_{n-1}^{\alpha} \in \dot{G}\right\}$ and $\forall \alpha \in \dot{X}_{n}\left(\dot{f}_{n}(\alpha)<\dot{f}_{n-1}(\alpha)\right)$ ".

For each $n<\omega$ and $\alpha \in E_{n+1}, q_{n+1}^{\alpha} \leqslant p_{n+1}$, hence $q_{n+1}^{\alpha} \|_{\bar{P}} " \alpha \in \dot{X}_{n+1} \subseteq$ $\left\{\alpha \in B_{n} \mid q_{n}^{\alpha} \in \dot{G}\right\}$ and $g_{n+1}(\alpha)=\dot{f}_{n+1}(\alpha)<\dot{f}_{n}(\alpha)$ ", and so $\alpha \in B_{n} \subseteq E_{n}, q_{n+1}^{\alpha} \leqslant q_{n}^{\alpha}$ and by $(2), g_{n+1}(\alpha)<g_{n}(\alpha)$. Hence $B_{n+1} \|_{\overline{R(I)}}{ }^{~ "} \delta_{n+1}=\left[g_{n+1}\right]<\left[g_{n}\right]=\delta_{n}$ ", yielding a strictly decreasing sequence of ordinals $\left\langle\delta_{n}\right| n\langle\omega\rangle$; contradiction.

Unlike the game $G_{1}(I), G(I)$ is not determined (see for instance [4, Theorem 1]), and indeed in contrast to precipitousness the property "Player Two has a winning strategy in $G(I)$ " behaves rather badly in forcing extensions. $I$ is said to be atomless iff for each $A \in I^{+}$there are disjoint sets $A_{0}, A_{1} \in P(A) \cap I^{+}$.

Theorem 3.3. Suppose $I$ is atomless and $P$ is the forcing notion for adding a Cohen subset of $\omega$, then $\|_{P}$ "Player Two does not have a winning strategy in $G(J)$ "'.

First we need the following

Lemma 3.4. If $|P|<\kappa, p \in P$ and $\dot{X}$ is a $P$ name such that $p \|_{\bar{P}}$ " $\dot{X} \in J^{+}$", then there is an $A \in I^{+}$and $a q \leqslant p$ such that $q \|_{\Gamma^{P}}$ " $A \subseteq \dot{X}$ ".

Proof. As in Theorem 1.5 there is an $E \in I^{+}$and $\left\{q^{\alpha} \mid \alpha \in E\right\} \subseteq P$ such that for each $\alpha \in E, q^{\alpha} \leqslant p$ and $q^{\alpha} \|_{\bar{P}}$ " $\alpha \in \dot{X}$ ". By $\kappa$-completeness there is a $q \leqslant p$ such that $A=\left\{\alpha \in E \mid q^{\alpha}=q\right\} \in I^{+}$, and hence $q \|_{\bar{P}}{ }^{\prime} A \subseteq \dot{X} "$.

Proof of Theorem 3.3. $P=\{p \mid p$ is a function with $\operatorname{dom}(p) \in \omega$ and $\operatorname{ran}(p) \subseteq 2\}$, ordered by $p \leqslant q$ iff $p \supseteq q$. Suppose $p \in P$ and $\dot{F}$ is a $P$ name such that $p \|_{\bar{P}}$ " $\dot{F}$ is a positional winning strategy for Player Two in $G(J)$ ". We are taking the strategy to be positional (i.e. to depend only on the last move of the opponent) for notational covenience only. It will be clear that what follows could be proved without this additional assumption.

We define a $P$ name $\dot{f}$ of the form $\dot{f}=\left\{\left\langle\left\langle n, A_{n}^{\delta}\right\rangle, p_{n}^{\delta}\right\rangle \mid n<\omega, \delta<\delta_{n}\right\}$ such that for each $n<\omega$

(1) $W_{n}=\left\{p_{n}^{\delta} \mid \delta<\delta_{n}\right\}$ is a maximal antichain below $p$ in $P$, and for each $\delta<\delta_{n}$, $\operatorname{dom}\left(p_{n}^{\delta}\right) \geqslant n$ and $A_{n}^{\delta} \in I^{+}$

(2) $\forall \beta<\delta_{n+1} \exists \delta<\delta_{n}\left(p_{n+1}^{\beta} \leqslant p_{n}^{\delta}\right)$. i.e. $W_{n+1}$ refines $W_{n}$

(3) if $\beta<\delta_{n+1}, \delta<\delta_{n}$ and $p_{n+1}^{\beta} \leqslant p_{n}^{\delta}$ then $p_{n+1}^{\beta} \|{ }_{\bar{P}}{ }^{~} A_{n+1}^{\beta} \subseteq \dot{F}\left(A_{n}^{\delta}\right) \subseteq A_{n}^{\delta}$ ". 
We construct $\dot{f}\left\lceil m=\left\{\left\langle\left\langle n, A_{n}^{\delta}\right\rangle, p_{n}^{\delta}\right\rangle \mid n<m, \delta<\delta_{n}\right\}\right.$ by induction on $m$; $\delta_{0}=1, p_{0}^{0}=p$ and $A_{0}^{0}=\kappa$, hence suppose we have $\dot{f} \uparrow(m+1)$ satisfying (1), (2) and (3). For each $\delta<\delta_{m}, p_{m}^{\delta} \leqslant p$ and $A_{m}^{\delta} \in I^{+}$, hence $p_{m}^{\delta} \|_{\bar{P}}{ }^{"} \dot{F}\left(A_{m}^{\delta}\right) \in$ $P\left(A_{m}^{\delta}\right) \cap J^{+}$", and so by Lemma 3.4 we may find a maximal antichain $T_{m}^{\delta}$ below $p_{m}^{o}$ in $P$ and sets $\left\{B_{q} \mid q \in T_{m}^{\delta}\right\} \subseteq I^{+}$such that for each $q \in T_{m}^{\delta}, q \|_{\bar{P}}$ " $B_{q} \subseteq \dot{F}\left(A_{m}^{\delta}\right)$ ". Since $I$ is atomless, for each $q \in T_{m}^{o}$ there are disjoint sets $B_{q}^{0}, B_{q}^{1} \in P\left(B_{q}\right) \cap I^{+}$, and so for each $\left.i<2, q^{\wedge} i=q \cup\{\operatorname{dom}(q), i\rangle\right\} \|{ }_{\bar{P}}{ }^{~ "} B_{q}^{i} \subseteq$ $\dot{F}\left(A_{m}^{\delta}\right)$ " and $\operatorname{dom}\left(q^{\wedge} i\right) \geqslant \operatorname{dom}\left(p_{m}^{\delta}\right)+1 \geqslant m+1$. Hence we may define $\dot{f} \uparrow(m+2)=\dot{f} \uparrow(m+1) \cup\left\{\left\langle\left\langle m+1, B_{q}^{i}\right\rangle, q \wedge i\right\rangle \mid i<2, q \in T_{m}^{\delta}\right.$ for some $\left.\delta<\delta_{m}\right\}$ such that (1), (2) and (3) continue to hold.

Clearly $p \|_{\bar{P}}$ " $\dot{f}(0), \dot{F}(\dot{f}(0)), \dot{f}(1), \dot{F}(\dot{f}(1)), \cdots$ is a play of $G(J) "$, and hence since $\dot{F}$ is assumed to be winning there is an $s \leqslant p$ and an $\alpha<\kappa$ such that $s \|_{\bar{P}}$ " $\forall n<\omega, \alpha \in \dot{f}(n)$ ". Pick $n \geqslant \operatorname{dom}(s)$ and $\delta<\delta_{n}$ such that $s$ and $p_{n}^{\delta}$ are compatible, then since $\operatorname{dom}\left(p_{n}^{\delta}\right) \geqslant n$ we must have $p_{n}^{\delta} \leqslant s$. But now if $q \in T_{n}^{\delta}$ there is a $j<2$ such that $\alpha \notin B_{q}^{j}$, and hence $q \wedge j \|_{\bar{P}}$ " $\dot{f}(n+1)=B_{q}^{j}$ and $\alpha \notin B_{q}^{j}$, contradicting $q^{\wedge} j \leqslant q \leqslant p_{n}^{\delta} \leqslant s$.

Of course it is crucial that our ideal in Theorem 3.3 should be atomless for, as is well known, if $I$ is prime and $|P|<\kappa$ then $\|_{P}$ " $J$ is prime", and in particular $\|_{\bar{P}}$ "Player Two has a winning strategy in $G(J)$ ".

As for preservation, Lemma 3.4 easily yields the following (rather weak) result whose proof we leave to the reader.

Theorem 3.5. Suppose $|P|<\kappa$ and $P$ is $(\omega, \infty)$-distributive, then if Player Two has a winning strategy in $G(I), \|_{\bar{P}}{ }^{~ " P l a y e r ~ T w o ~ h a s ~ a ~ w i n n i n g ~}$ strategy in $G(J)$ ".

Also, Galvin, Jech and Magidor [4] proved that if $I$ is normal and prime and $P$ is the Levy collapse for making $\kappa$ into $\omega_{2}$, then $\|\left.\right|_{P}{ }^{~ "} R(J)$ has a dense $\omega$-closed subset (and in particular, Player Two has a winning strategy in $G(J))$ '". The following theorem strengthens this result, (indeed the proof is similar) and also improves a result of Kanai [11, Corollary 2]. $P$ is said to be $\mu$-directed closed iff whenever $Q \subseteq P$ is directed with $|Q|<\mu$, there is a $p \in P$ such that $p \leqslant q$ for each $q \in Q$.

Theorem 3.6. Suppose $\mu<\kappa, I$ is prime and $P$ has the $\kappa$-chain condition and is $\mu$-directed closed. Then $\|_{\bar{P}}{ }^{"} R(J)$ has a dense $\mu$-directed closed subset".

Proof. Suppose $G$ is $P$ - $V$-generic and $X \in J_{G}^{+}$. As in Theorem 3.2 we 
may find a $P$ name $\dot{X}$ for $X$, conditions $p \geqslant s \geqslant r \in G, E \in I^{+}\left(=I^{*}\right)$ and $\left\langle q^{\alpha} \mid \alpha \in E\right\rangle \in V$ such that for each $\alpha \in E, q^{\alpha} \leqslant s, q \|_{\bar{P}}$ " $\alpha \in \dot{X}$ " and $r \|_{\bar{P}}$ " $\left\{\alpha \in E \mid q^{\alpha} \in \dot{G}\right\} \in J^{+} "$. Hence $F(X)=\left\{\alpha \in E \mid q^{\alpha} \in G\right\} \in P(X) \cap J_{G}^{+}$and $Q=$ $\left\{F(X) \mid X \in J_{G}^{+}\right\}$is dense in $R\left(J_{G}\right)$. The proof that $Q$ is $\mu$-directed closed is easy and we leave the details to the reader).

\section{REFERENCES}

[1] J. Baumgartner and A. Taylor, Saturation properties of ideals in generic extensions II, Trans. Amer. Math. Soc., 271 (1982), 587-609.

[2] J. Baumgartner, A. Taylor and S. Wagon, Structural properties of ideals, Dissertationes Mathematicae, vol. 197 (1982).

[ 3 ] M. Foreman, Games played on Boolean algebras, J. Symbolic Logic, 48 (1983), $714-723$.

[4] F. Galvin, T. Jech and M. Magidor, An ideal game, J. Symbolic Logic, 43 (1978), 284-291.

[5] T. Jech, Some properties of $\kappa$-complete ideals defined in terms of infinite games, Ann. Pure Appl. Logic, 26 (1984), 31-46.

[6] T. Jech and K. Prikry, Ideals over uncountable sets, Mem. Amer. Math. Soc., 18 (2) (1979).

[ 7 ] C. A. Johnson, Distributive ideals and partition relations, J. Symbolic Logic, to appear.

[ 8 ] - Some properties of precipitous and related ideals, Ph.D. Thesis, Leeds University (1984).

[ 9 ] Y. Kakuda, Saturated ideals in Boolean extensions, Nagoya Math. J., 48 (1972), $159-168$.

[10] - On a condition for Cohen extensions which preserve precipitous ideals, J. Symbolic Logic, 46 (1981), 296-300.

[11] Y. Kanai, On quotient algebras in generic extensions, Comm. Math. Univ. Sancti Pauli, 33 (1984), 71-77.

[12] M. Magidor, Precipitous ideals and $\Sigma_{4}^{\prime}$ sets, Israel J. Math., 35 (1980), 109-134.

Mathematics Department

Keele University

Keele, Staffs. ST5 5BG

England 